\title{
Comparaciones basadas en biomarcadores de crudos extraídos de la Formación Springhill (Cuenca Austral) y de la Formación Bajo Barreal (Cuenca Golfo San Jorge), Argentina
}

\section{Biomarker-based comparisons of crude oil extracted from the Springhill Formation (Austral Basin) and the Bajo Barreal Formation (Golfo San Jorge Basin), Argentina}

\author{
Germán Javier Tomas ${ }^{1,2, ~}{ }^{*}$, Adrián Javier Acuña ${ }^{2}$ \\ ${ }^{1}$ Centro de Investigación y Transferencia de la Provincia de Santa Cruz, Río Gallegos, Argentina. ORCID ID: https://orcid. \\ org/0000-0001-7836-4005 \\ ${ }^{2}$ Facultad Regional Santa Cruz, Universidad Tecnológica Nacional, Río Gallegos, Argentina. ORCID ID: https://orcid.org/0000- \\ 0002-0382-3681 \\ *Corresponding author: germanjaviertomas@frsc.utn.edu.ar
}

\section{RESUMEN}

En Argentina existen varias cuencas productivas de petróleo, entre ellas las Cuencas Golfo San Jorge y Austral. Sus sucesiones estratigráficas contienen varias unidades que actúan como rocas reservorio, destacando en este estudio las formaciones Bajo Barreal (98 Ma) y Springhill (134 Ma). El objetivo de este trabajo fue comparar crudos extraídos de estas formaciones usando biomarcadores geoquímicos orgánicos: saturados (n-parafinas, isoprenoides acíclicos pristano y fitano, esteranos y terpanos) y aromáticos (fenantrenos y dibenzotiofenos) para establecer similitudes y diferencias respecto al tipo de materia orgánica que les dio origen, el grado de biodegradación alcanzado por los mismos y, por otro lado, determinar la litología y las condiciones de depósito que controlaron la acumulación y preservación de la materia orgánica en sus rocas madre. Los crudos fueron sometidos a una separación de sus fracciones alifáticas y aromáticas por cromatografía de adsorción sólidolíquido en columna y cada uno de estos extractos obtenidos fue analizado mediante cromatografía de gases acoplada a espectrometría de masas (GC-MS). A partir de las relaciones de diagnóstico, calculadas con las abundancias relativas de los biomarcadores, se sugiere para los crudos de la Formación Springhill que la materia orgánica precursora fue de tipo mixto marino - continental, depositada en un ambiente con concentración moderada de oxígeno (disóxico-subóxico), en el cual la roca madre se caracterizó por ser de naturaleza marina siliciclástica, y no se observó biodegradación de estos. Por otro lado, los crudos asociados a la Formación Bajo Barreal son de tipo algal-lacustre severamente biodegradados, sedimentados en condiciones de anoxia en una roca generadora tipo carbonática. Estos resultados concuerdan con estudios previos realizados sobre crudos

Recibido el 27 de julio de 2021; Aceptado el 10 de diciembre de 2021; Publicado online el 07 de marzo de 2022

Citation / Cómo citar este artículo: Tomas, G.J. et al. (2021). Comparaciones basadas en biomarcadores de crudos extraídos de la Formación Springhill (Cuenca Austral) y de la Formación Bajo Barreal (Cuenca Golfo San Jorge), Argentina. Estudios Geológicos 78(1): e143. https://doi.org/10.3989/egeol.44405.606

Copyright: @ 2022 CSIC. Este es un artículo de acceso abierto distribuido bajo los términos de la licencia de uso y distribución Creative Commons Reconocimiento 4.0 Internacional (CC BY 4.0). 
provenientes de otros yacimientos de ambas formaciones, pero lo novedoso radica en que estas localizaciones nunca fueron estudiadas.

Palabras clave: Formación Springhill; Formación Bajo Barreal; Cuenca Austral; Cuenca Golfo San Jorge; biomarcadores; crudo

\section{ABSTRACT}

In Argentina, there are several crude oils-producing basins, including the Golfo San Jorge and Austral basins. Their stratigraphic columns present several units that behave as reservoir rocks, the Bajo Barreal and Springhill formations stand out in this study. The objective of this work was to compare crude oils extracted from these formations using the organic geochemical biomarkers: saturated (n-paraffins, acyclic isoprenoids pristane and phytane, steranes and terpanes) and aromatic (phenanthrenes and dibenzothiophenes), with the purpose of evaluating similarities and differences in order to establish the crude oil precursor organic matter type, the biodegradation degree and, the lithology and depositional conditions that controlled the accumulation and preservation of organic matter in theirs source rock. These crude oils were subjected to separation of their aliphatic and aromatic fractions by solid-liquid adsorption and column analyzed each one by gas chromatography-mass spectrometry (GC-MS). From the diagnostic ratios, calculated from the relative abundances of the biomarkers, it is suggested for the Springhill Formation crude oils that the organic matter source (mixed marine-continental), were deposited in a sedimentary environment with moderate-to-low oxygen concentration (disoxyc-suboxyc), in which the source rock was characterized as shelf marine shales and no biodegradation was observed. On the other hand, the crude oils associated with the Bajo Barreal Formation can be classified as a severely biodegraded algal-lacustrine type, deposited in anoxic conditions in a carbonate source rock. These results are in agreement with previous studies carried out on crudes from other reservoirs in both formations, but the novelty is that these locations have never been studied.

Keywords: Springhill Formation; Bajo Barreal Formation; Austral basin; Golfo San Jorge basin; biomarkers; crude oil

\section{Introducción}

En el petróleo existe un grupo de moléculas que poseen la capacidad de caracterizar genéticamente a los crudos que provienen de yacimientos petrolíferos. Las mismas se asocian a precursores definidos, es decir, permiten trazar una serie de reacciones químicas para conocer la molécula biológica de la cual derivan (Peters et al., 2005). En base a estos dos conceptos reciben el nombre de biomarcadores y desde 1970 se utilizan como una herramienta para los análisis dentro de la geoquímica orgánica (Speers \& Whitehead, 1969; Seifert \& Moldowan, 1981). Estos marcadores biológicos permiten establecer correlaciones entre crudos, entre crudos y roca madre, determinar su madurez térmica y el grado de biodegradación de los crudos analizados (Peters \& Moldowan, 1993). También son de utilidad para determinar el origen biológico de la materia orgánica, las condiciones ambientales en las que esta sedimentó (óxicas o anóxicas, marinas o continentales), y para conocer la litología de la roca generadora (Killops \& Killops, 2005). La concentración de biomarcadores en el petróleo es baja (ppm-ppb), sin embargo, pue- den ser detectados y caracterizados mediante el uso de la espectrometría de masas acoplada a la cromatografía de gases (GC-MS) (Stashenko et al., 2014).

El pristano $(\mathrm{P}=2,6,10,14$-tetrametilpentadecano $)$ y el fitano $(F=2,6,10,14$-tetrametilhexadecano) son biomarcadores muy estudiados debido a la facilidad con la que se determinan por las técnicas analíticas convencionales. El pristano se forma por la degradación diagenética del fitol (molécula derivada de la clorofila), a través de un proceso de oxidación y el fitano debe generarse a través de un proceso de reducción, por lo que la relación $\mathrm{P} / \mathrm{F}$ da indicios de las condiciones oxirreductoras de depositación (Didyk et al., 1978), sin embargo, es importante destacar que la madurez térmica condiciona esta relación (Peters et al., 2005). Además, son muy importantes en la interpretación geoquímica de las muestras de crudos debido a que su concentración es la más elevada respecto del resto de los otros hidrocarburos isoprenoides acíclicos presentes en esta fracción (Peters et al., 2005). Por otro lado, la relación pristano/heptadecano $\left(\mathrm{P} / \mathrm{n}-\mathrm{C}_{17}\right)$ en función de fitano/octadecano $\left(\mathrm{F} / \mathrm{n}-\mathrm{C}_{18}\right)$ se utiliza para sugerir el tipo de materia orgánica precursora (Shanmugam, 1985). 
Los compuestos biológicos que conservan mejor su estructura a lo largo de la diagénesis y catagénesis son los hopanoides y terpenoides, precursores de los hopanos y terpanos tricíclicos. Debido a esto, se consideran entre los más importantes del petróleo para determinar el tipo de materia orgánica que le dio origen (Hunt, 1996). Los hopanoides y terpenoides se encuentran en la membrana celular de las células procariotas y de las algas, respectivamente (Philp, 1985). Entre los hopanos debe tenerse en cuenta al $17 \alpha, 21 \beta(\mathrm{H})$ hopano $\mathrm{C}_{30}\left(\mathrm{H}_{30}\right)$ ya que generalmente es el dominante en las muestras de crudo, su relación con el gammacerano $\left(\mathrm{G}_{30}\right)$ definida como $\left(\mathrm{G}_{30} / \mathrm{H}_{30}\right) \mathrm{x}$ 100 es un indicador de la salinidad presente en el medio (Seifert et al., 1984). Además, el gammacerano también se considera como un indicador de anoxia por estratificación de la columna de agua (Shinningue-Damste 1995; Peters et al., 2005). Otros biomarcadores de interés son los esteranos, los cuales no existen como tales en los organismos vivos. Estos se encuentran como esteroles en organismos eucariotas como algas, animales y plantas superiores por lo que también proporcionan información sobre la materia orgánica precursora (Mackenzie et al., 1982). Las arcillas catalizan la transformación de esteranos a diasteranos durante la maduración térmica y una relación entre estos refleja el contenido de arcilla presente en una roca madre (Lerch et al., 2016).

En los estudios geoquímicos también se analizan moléculas con un origen inespecífico conocidas como marcadores aromáticos que complementan la información suministrada por los biomarcadores (Killops \& Killops, 2005). Entre ellas, la relación de diagnóstico del dibenzotiofeno (DBT) con el fenantreno $(\mathrm{Ph})$ representada en función de $\mathrm{P} / \mathrm{F}$ es usada para sugerir la litología de la roca generadora (Hughes et al., 1995). El objetivo de este trabajo fue comparar crudos extraídos de las Formaciones Springhill y Bajo Barreal usando biomarcadores como parámetro de análisis para establecer similitudes y diferencias respecto del tipo de materia orgánica que les dio origen, las condiciones de depósito que controlaron la acumulación y preservación de la materia orgánica, la litología de sus rocas madre respectivas y el grado de biodegradación. La originalidad de este trabajo radica en el estudio de crudos que provienen de pozos y/o yacimientos no documentados hasta la fecha que contribuyen a respaldar o refutar los resultados publicados anteriormente.

\section{Marco Geológico}

Las cuencas de estudio, Austral y Golfo San Jorge están ubicadas en la porción meridional de la Patagonia (Figura 1). La Cuenca Austral se formó durante el Triásico-Jurásico y comprende una superficie de $146.000 \mathrm{~km}^{2}$ en el extremo sudoeste de la placa sudamericana. Abarca las provincias argentinas de Santa Cruz y Tierra del Fuego, parte de la plataforma continental argentina, el estrecho de Magallanes y la provincia chilena Magallanes (Aramendia et al., 2018). Hacia el norte limita contra el macizo del Deseado, un alto estructural emplazado en el centro-norte de la provincia de Santa Cruz; hacia el noroeste se conecta con la parte occidental de la Cuenca Golfo San Jorge la cual se sitúa entre el norte de la provincia de Santa

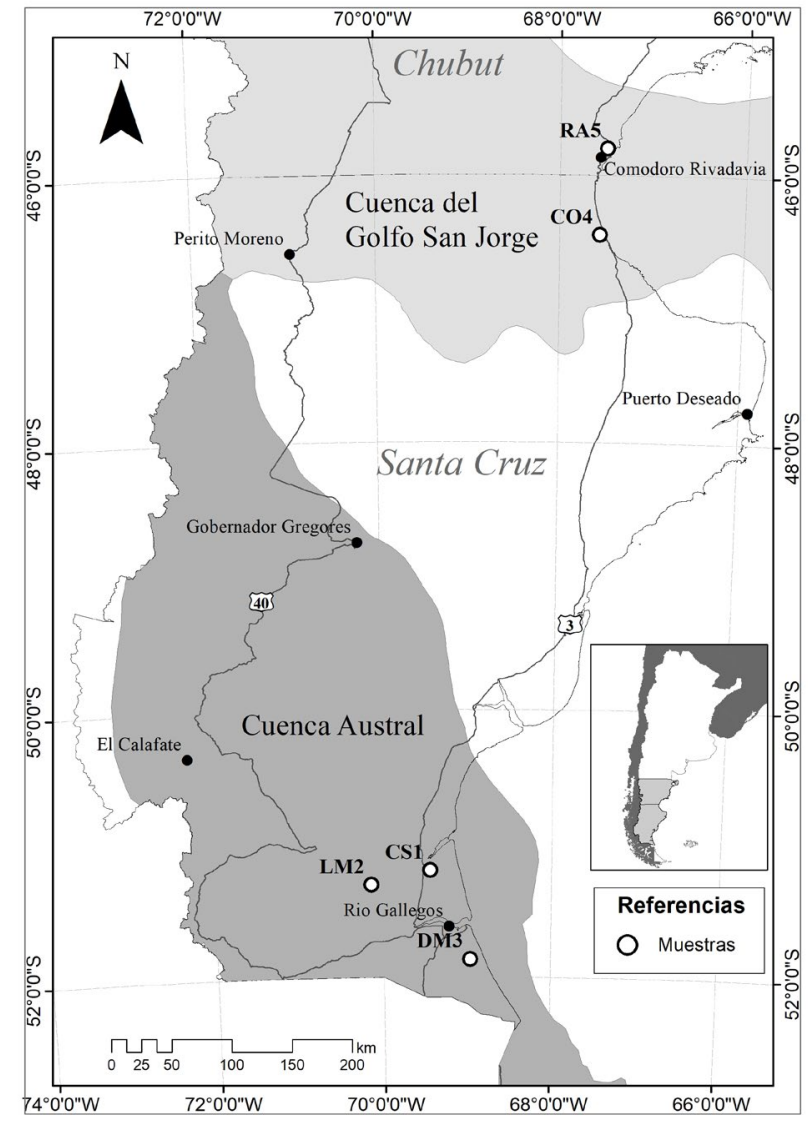

Figura 1.- Mapa que ubica los yacimientos donde se han tomado las muestras de crudo bajo estudio (CS1, LM2, DM3, CO4 y RA5) en las Cuencas Austral y Golfo San Jorge, en el sur de la Patagonia, Argentina. 
Cruz y el sur de la provincia de Chubut (Cuitiño et al., 2019).

El basamento de la Cuenca Austral conocido como Formación Tobífera (140 Ma) está formado por espesas secuencias volcaniclásticas ácidas (riolitas e ignimbritas) y depósitos lacustres (Barredo et al., 2012). Por encima, suprayace una roca yacimiento formada por areniscas glauconíticas, de cuarzo y lutitas, depositadas en un entorno marino conocida como Formación Springhill (134 Ma, Mpodozis et al., 2011). Luego le sigue la Formación Inoceramus Inferior (122 Ma, roca madre) con la cual forman el sistema petrolero más importante de la cuenca ya que, desde la década de los cuarenta del siglo XX, aportaron prácticamente la totalidad de las reservas hidrocarburíferas descubiertas en la región
(Ramos et al., 2019). Este sistema se caracterizó por una migración lateral de los hidrocarburos de media a larga distancia (decenas de kilómetros) desde la roca generadora "Inoceramus Inferior" hacia el reservorio "Springhill" cargando la zona de talud y plataforma estable, donde se desarrollan las principales acumulaciones (Zerfass et al., 2017). Las formaciones Margas Verdes (112 Ma) y Magallanes (77 Ma) forman el segundo sistema petrolero en importancia dentro de la Cuenca Austral debido al volumen de hidrocarburos entrampados en el mismo. Estas reservas de gran importancia para la provincia de Santa Cruz comenzaron a explotarse en los años 90 del siglo XX (Richiano et al., 2016). El petróleo generado en la Formación Margas Verdes (roca madre) migró verticalmente hacia la trampa de

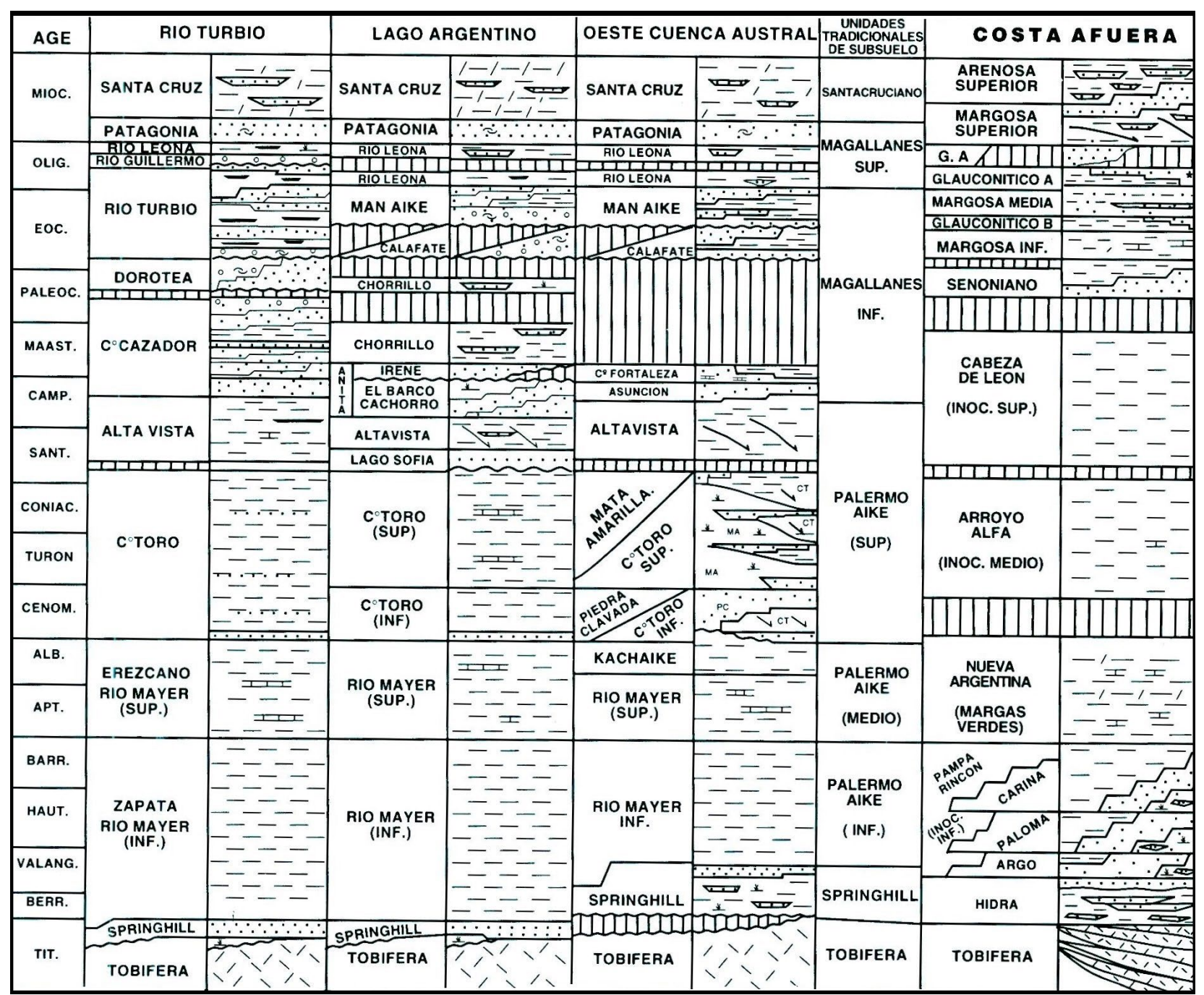

Figura 2.- Diagrama estratigráfico con comparación de las diferentes localidades de la Cuenca Austral. Adaptado de Schiuma et al. (2018). 
hidrocarburos en el Miembro Inferior de la Formación Magallanes (77 Ma) a través de un sistema de corta distancia dado por un conjunto de fallas cuasi verticales (Schiuma et al., 2018). En la Figura 2 se observa un diagrama estratigráfico de la Cuenca Austral para diferentes localidades de esta en el cual se observan las unidades tradicionales del subsuelo.

La Cuenca Golfo San Jorge, ubicada en la porción central de la Patagonia, es la más antigua y prolífica productora de hidrocarburos de Argentina. Se extiende entre los paralelos $45^{\circ}$ y $47^{\circ}$ Sur y los meridianos $65^{\circ}$ y $71^{\circ}$ Oeste abarcando porciones de las provincias de Chubut y Santa Cruz sobre una superficie estimada de $180.000 \mathrm{~km}^{2}$ (Ramos, 2015). En esta cuenca coexisten espacialmente al menos dos tipos de secuencias sedimentarias evolutivamente diferenciables (conocidas como Neocomiana y Chubutiana en sentido amplio, aunque sus rangos temporales son aún inciertos), y

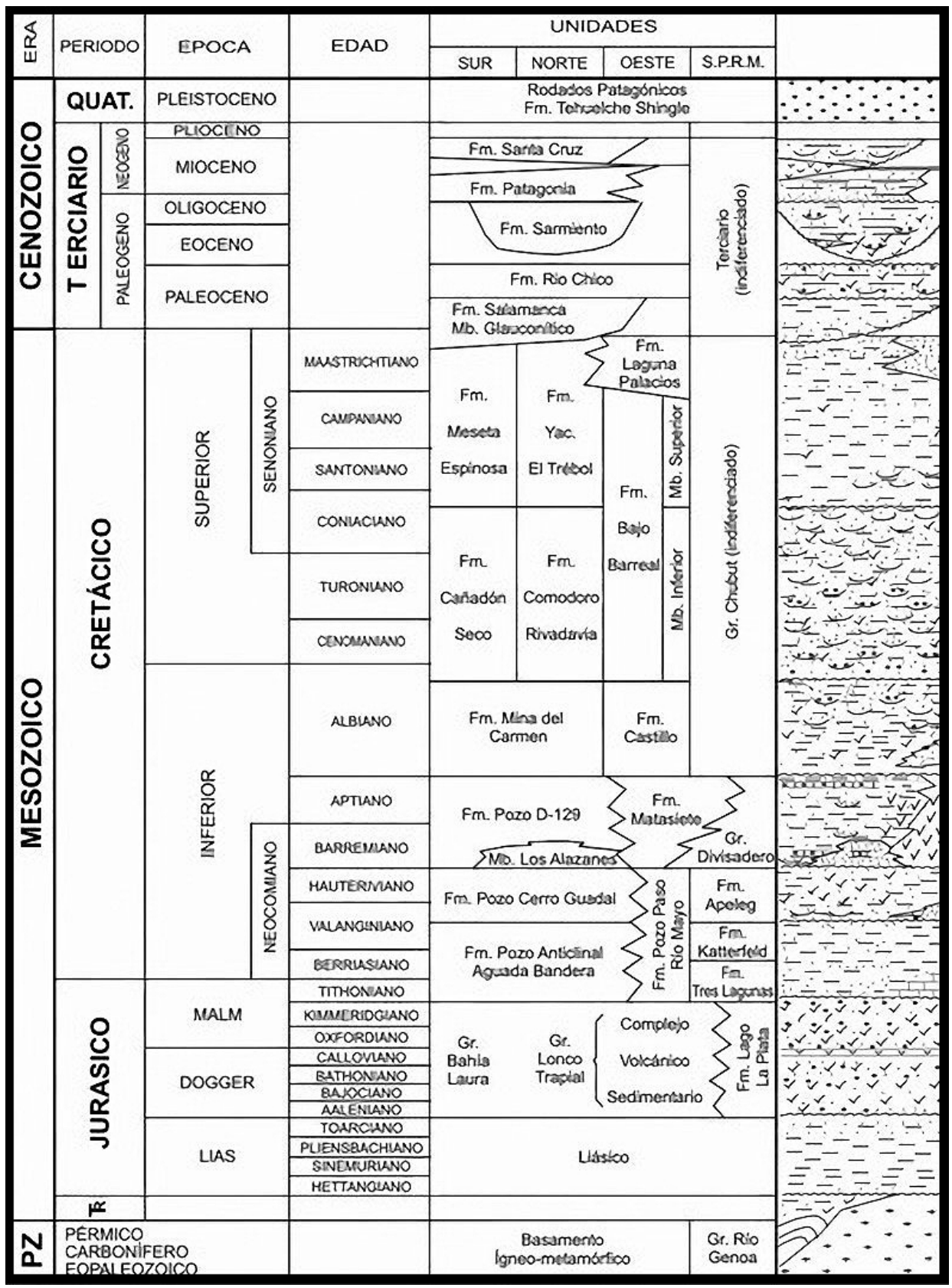

Figura 3.- Diagrama estratigráfico de la Cuenca Golfo San Jorge. Adaptado de Figari et al. (1999). 
que poseen un registro sedimentario, control tectónico y geométrico particular (Figari et al., 2002). Esta ha sido la causa determinante de la existencia de dos sistemas petroleros principales, el más productivo de ellos conocido como Pozo D-129/Bajo Barreal se extiende ampliamente en el ámbito centro-oriental de la cuenca y comprende todos los yacimientos de los Flancos Norte, Sur y la Faja Plegada (Laffitte et al., 2001). La roca yacimiento es la Formación Bajo Barreal (98 $\mathrm{Ma}$ ), unidad que supera $\operatorname{los} 2.000 \mathrm{~m}$ de espesor en el depocentro chubutiano y menos de $400 \mathrm{~m}$ en los bordes (Hechem et al., 1990). Esta ha sido abastecida por la roca generadora Formación Pozo D-129 (124 Ma), la cual presenta varias secuencias compuestas de pelitas negras con altos contenidos orgánicos que proveyeron una formidable fuente de hidrocarburos para la cuenca (Sylwan, 2001). En su período de depositación se acepta la presencia de un gran lago estratificado, relativamente somero, con fluctuaciones en los grados de hipersalinidad/alcalinidad (Gómez Omil et al., 1990). Por otro lado, el sistema petrolero Aguada Bandera/ Bajo Barreal, que incluye una porción menor de las reservas, se restringe al Flanco Occidental y parcialmente al borde suroeste de la Faja Plegada (Figari et al., 1999). Los contenidos orgánicos de esta roca madre (150 Ma) se sitúan en torno al $2 \%$ de COT, el kerógeno es predominantemente amorfo y está asociado con abundantes restos de algas Celyphus rallus (entorno lacustre distal, columna de agua estratificada). Los crudos asociados son ricos en parafina y muestran una mayor influencia terrestre que los vinculados al otro sistema fuente Pozo D-129 (Laffitte \& Hechem, 1993). Finalmente, existe una tercera unidad como roca madre dentro de la cuenca conocida como Formación Pozo Cerro Guadal (133 Ma). La misma se caracteriza por organofacies terrígenas y una distribución de terpanos tri y tetracíclicos que permite diferenciarla de las otras dos formaciones (Sylwan, 2008). En la Figura 3 se observa la columna estratigráfica generalizada de la Cuenca Golfo San Jorge.

\section{Metodología}

\section{Muestras}

Se analizaron cinco muestras de crudo, tres pertenecientes a la Formación Springhill y dos a la Formación Pozo D-129. Los crudos de la Cuenca Austral denominados CS1, LM2 y DM3 se obtuvieron de los yacimientos Cañadón Salto, La Maggie y Del Mosquito a 1300,1500 y $1300 \mathrm{~m}$ de profundidad, respectivamente. Por otro lado, los crudos de la Cuenca Golfo San Jorge denominados CO4 y RA5 se obtuvieron de los reservorios Cañadón Seco y Restinga Alí a 1200 y $1600 \mathrm{~m}$ de profundidad, respectivamente. Los crudos fueron recolectados y transportados al laboratorio en botellas limpias de vidrio (un litro de capacidad color ámbar) para evitar la fotooxidación. En el llenado de las botellas, se evitó la presencia de cámara de aire para minimizar el impacto del oxígeno en la estabilidad de las muestras y prevenir su biodegradación. Las muestras fueron almacenadas en lugares oscuros y secos a temperatura ambiente hasta la realización de los análisis dentro de las siguientes $72 \mathrm{~h}$ en el Laboratorio de Química de la Facultad Regional Santa Cruz (Universidad Tecnológica Nacional).

\section{Acondicionamiento de muestras de crudo}

Teniendo en consideración trabajos tales como Leal et al. (2011) y Tomas et al. (2020), el procedimiento para la obtención de las fracciones a analizar consistió en la separación de los crudos por cromatografía de adsorción sólido-líquido en columna, en hidrocarburos saturados, compuestos aromáticos y resinas-asfáltenos. Las muestras se sometieron a una separación en columna de vidrio $(20 \mathrm{~cm} \times 1,2 \mathrm{~cm})$ empacadas con $3 \mathrm{~g}$ de gel de sílice activado, al que se le adicionó $50 \mathrm{mg}$ de sulfato de sodio activado y $50 \mathrm{mg}$ de alúmina activada en su parte superior. Se sembraron aproximadamente $100 \mu 1$ de crudo en la columna, luego se eluyeron sucesivamente con $10 \mathrm{ml}$ de n-pentano y $10 \mathrm{ml}$ de diclorometano, para obtener los extractos de hidrocarburos saturados (fracción alifática) y compuestos aromáticos (fracción aromática), respectivamente. Ambas fracciones se concentraron por separado hasta $0,5 \mathrm{ml}$ bajo corriente de nitrógeno, se transfirieron a un vial, y se almacenaron $\mathrm{a}-15^{\circ} \mathrm{C}$ hasta su análisis, quedando al final las fracciones de resinas y asfáltenos, retenidas en la columna.

\section{Análisis cromatográfico}

En base a las publicaciones de Stashenko et al. (2014) y Tomas et al. (2020), por cada muestra de 
Tabla 1.- Parámetros moleculares derivados de los biomarcadores y marcadores aromáticos seleccionados en las muestras de crudo bajo análisis.

\begin{tabular}{|c|c|c|c|c|c|}
\hline \multirow[t]{2}{*}{ Relación de Diagnóstico } & \multicolumn{3}{|c|}{$\begin{array}{c}\text { Cuenca Austral } \\
\text { Formación Springhill }\end{array}$} & \multicolumn{2}{|c|}{$\begin{array}{l}\text { Cuenca Golfo San Jorge } \\
\text { Formación Bajo Barreal }\end{array}$} \\
\hline & cs1 & LM2 & DM3 & $\mathrm{CO} 4$ & RA5 \\
\hline $\mathrm{P} / \mathrm{F}$ & 2,59 & 1,94 & 2,04 & 1,06 & 0,80 \\
\hline $\mathrm{P} / \mathrm{n}-\mathrm{C}_{17}$ & 0,54 & 0,70 & 0,48 & 0,40 & 1,66 \\
\hline $\mathrm{F} / \mathrm{n}-\mathrm{C}_{18}$ & 0,26 & 0,38 & 0,24 & 0,38 & 1,81 \\
\hline $\mathrm{n}-\mathrm{C}_{29} / \mathrm{n}-\mathrm{C}_{17}$ & 0,38 & 0,36 & 0,57 & 0,26 & 0,52 \\
\hline$\left(\mathrm{G}_{30} / \mathrm{H}_{30}\right) * 100$ & 0,34 & 0,48 & 0,26 & 13,6 & 14,1 \\
\hline$\% \mathrm{~S}_{27}$ & 50,1 & 55,5 & 45,4 & 24,2 & 26,9 \\
\hline$\% \mathrm{~S}_{28}$ & 21,9 & 17,3 & 14,0 & 31,9 & 29,1 \\
\hline$\% \mathrm{~S}_{29}$ & 28,0 & 27,2 & 30,6 & 43,9 & 44,0 \\
\hline $\mathrm{D}_{27} / \mathrm{S}_{27}$ & 1,19 & 1,17 & 1,02 & 0,42 & 0,30 \\
\hline $\mathrm{DBT} / \mathrm{Ph}$ & 0,20 & 0,27 & 0,09 & 0,04 & 0,09 \\
\hline
\end{tabular}

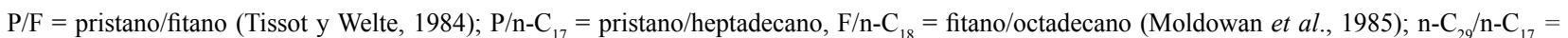
nonacosano/heptadecano; $\left(\mathrm{G}_{30} / \mathrm{H}_{30}\right) * 100=$ índice de gammacerano (Seifert et al., 1984); \% $\mathrm{S}_{27}=\left(\mathrm{S}_{27} / \mathrm{S}_{27}+\mathrm{S}_{28}+\mathrm{S}_{29}\right) * 100, \% \mathrm{~S}_{28}=\left(\mathrm{S}_{27} / \mathrm{S}_{27}+\mathrm{S}_{28}\right.$ $\left.+\mathrm{S}_{29}\right) * 100, \% \mathrm{~S}_{29}=\left(\mathrm{S}_{27} / \mathrm{S}_{27}+\mathrm{S}_{28}+\mathrm{S}_{29}\right) * 100$ (Peters et al., 2005); $\mathrm{D}_{27} / \mathrm{S}_{27}=$ diasteranos/colestanos; DBT/Ph = dibenzotiofeno/fenantreno (Hughes et al., 1995).

crudo se inyectó en un cromatógrafo de gases, un $\mu 1$ de los extractos alifáticos y aromáticos en modo Splitless. La separación cromatográfica se realizó en un cromatógrafo de gases Agilent modelo 7890A, con un detector de espectrometría de masas de la misma marca (modelo 5975C). Se utilizó una columna HP5ms de $30 \mathrm{~m}$ de largo, con un diámetro interno de $0,32 \mathrm{~mm}$ y un espesor de película de 0,25 $\mu \mathrm{m}$. La temperatura del inyector se ajustó a $290^{\circ} \mathrm{C}$ y se utilizó helio como gas acarreador con una velocidad de flujo de 1,2 ml. $\mathrm{min}^{-1}$. El programa de temperatura utilizado fue el siguiente: temperatura inicial de $55^{\circ} \mathrm{C}$ durante $2 \mathrm{~min}$, seguido de una rampa de $6^{\circ} \mathrm{C} \cdot \mathrm{min}^{-1}$ hasta alcanzar los $270^{\circ} \mathrm{C}$, pasando directamente a otra rampa de $3^{\circ} \mathrm{C} . \mathrm{min}^{-1}$ hasta alcanzar $300^{\circ} \mathrm{C}$, temperatura que se mantuvo durante $17 \mathrm{~min}$. El tiempo total de corrida fue de $65 \mathrm{~min}$. El detector de masas se utilizó con una temperatura de la fuente de iones y de la línea de transferencia de $230^{\circ} \mathrm{C}$ y $180^{\circ} \mathrm{C}$ respectivamente y una energía de impacto de $70 \mathrm{eV}$. El escaneo de masas entre 30 y 400 uma se realizó para obtener el fragmentograma del ion $\mathrm{m} / \mathrm{z}=$ 99 con el fin de determinar los isoprenoides acíclicos pristano y fitano y, además analizar la distribución de n-parafinas en las fracciones de hidrocarburos saturados de los crudos. Por su parte, el modo SIM
Scan (monitoreo de iones seleccionados) se usó para determinar los iones $\mathrm{m} / \mathrm{z}=178$ (fenantreno) y 184 (dibenzotiofeno) en las fracciones aromáticas, y los iones $\mathrm{m} / \mathrm{z}=191$ (terpanos) y 217 (esteranos), en los extractos alifáticos.

\section{Resultados}

Los resultados del análisis cromatográfico de las muestras se resumen en la Tabla 1 y se muestran en las Figuras 4 y 5. Los fragmentogramas de los crudos de la Cuenca Austral para el ion $\mathrm{m} / \mathrm{z}=99$ mostraron un patrón bimodal para los n-alcanos con ligeras diferencias entre ellos (Figura 4A, D y G). El crudo CO4 (Figura 4J) presentó una distribución tipo unimodal en el intervalo de $n-C_{12}$ a $n-C_{31}$, con un máximo en $\mathrm{n}-\mathrm{C}_{18}$. Finalmente, la otra muestra de la Cuenca Golfo San Jorge (Figura 4M) evidenció un levantamiento de la línea de base asociado a una mezcla compleja no resuelta (UCM) y una biodegradación incipiente (nivel 1-2 de la escala de biodegradación de Peters y Moldowan (1993)), principalmente por los comparativamente altos valores de las relaciones $\mathrm{P} / \mathrm{n}-\mathrm{C}_{17}$ y $\mathrm{F} / \mathrm{n}-\mathrm{C}_{18}$, respecto a los otros crudos, y la disminución relativa en la concentración de n-alcanos livianos. 


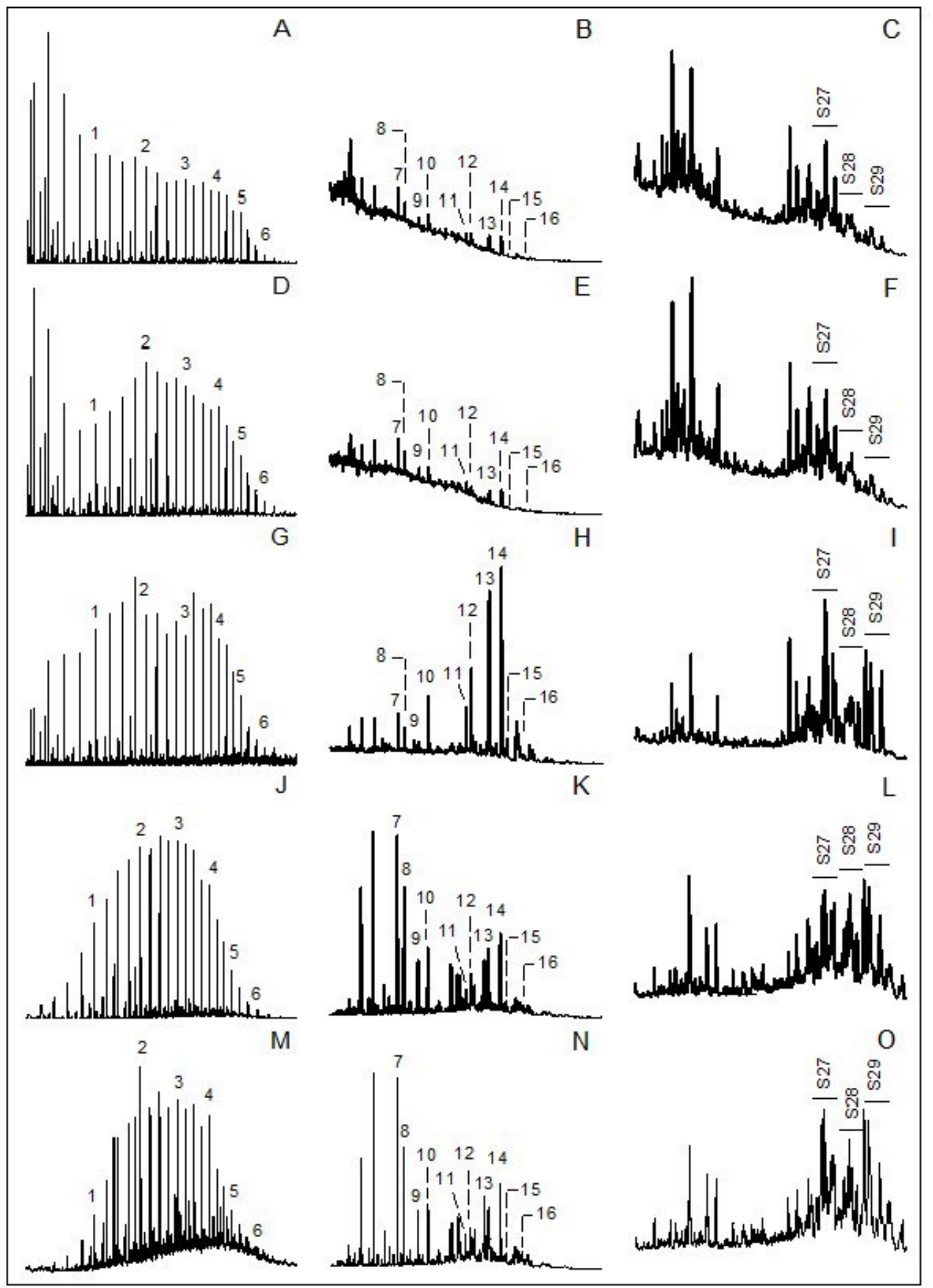

Figura 4.- Fragmentogramas de los iones $\mathrm{m} / \mathrm{z}=99,191$ y 217 de las muestras CS1 (A-C), LM2 (D-F), DM3 (G-I), CO4 (J-L) y RA5 (M-O). Los compuestos enumerados son los siguientes: 1- n-dodecano, 2- n-hexadecano, 3- n-eicosano, 4- n-tetracosano, 5n-heptacosano, 6- n-triacontano, 7- terpano tricíclico $C_{23}\left(T_{23}\right)$, 8- terpano tricíclico $C_{24}\left(T_{24}\right)$, 9- terpano tricíclico $C_{25}\left(T_{25}\right)$, 10- terpano tricíclico $\mathrm{C}_{26}\left(\mathrm{~T}_{26}\right)$, 11- trisnorneohopano (Ts), 12- trisnorhopano (Tm), 13- norhopano $\mathrm{C}_{29}\left(\mathrm{H}_{29}\right)$, 14- hopano $\mathrm{C}_{30}\left(\mathrm{H}_{30}\right)$, 15- moretano $\left(\mathrm{M}_{30}\right)$, 16- gammacerano $\left(G_{30}\right)$, colestanos $\left(S_{27}\right)$, ergostanos $\left(S_{28}\right)$ y estigmastanos $\left(S_{29}\right)$. 


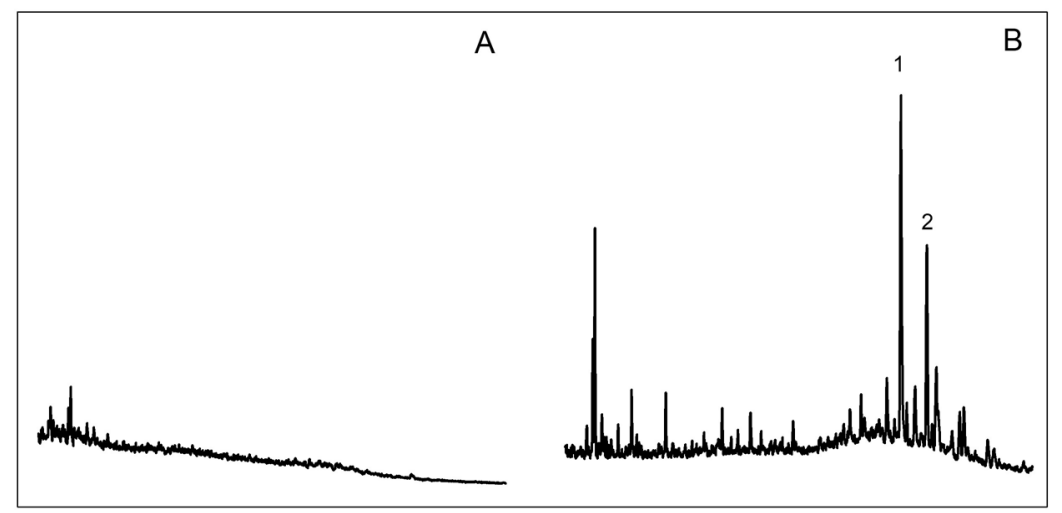

Figura 5.- Fragmentogramas del ion $\mathrm{m} / \mathrm{z}=177$ para las muestras CS1 (A) y RA5 (B) representativas de las Cuencas Austral y Golfo San Jorge, respectivamente. Los compuestos enumerados son los siguientes: 1 - hopano desmetilado $C_{28}$ y 2 - hopano desmetilado $C_{29}$.

Las distribuciones obtenidas a partir de los iones $\mathrm{m} / \mathrm{z}=191$ y 217 pusieron de manifiesto diferencias entre las muestras a nivel de cuencas. Los crudos CS1 y LM2 (Figuras 4B y E) se caracterizaron por una escasez de terpanos tanto tricíclicos como pentacíclicos (hopanos) y la prevalencia de los colestanos $\left(\mathrm{S}_{27}\right)$ frente a los otros esteranos regulares (Figuras 4C y F). En la muestra DM3 se aprecia que los hopanos (señales 12, 13, 14 y 15 estaban en mayor concentración respecto a los diterpanos tri- y tetracíclicos (Figura 4H) y a los colestanos (Figura 4I). A diferencia de los crudos anteriores, los petróleos CO4 y RA5, destacaron porque los diterpanos triy tetracíclicos (señales 7 y 8) presentaron mayores concentraciones que los hopanos (Figuras $4 \mathrm{~K}$ y N) y por tener a los estigmastanos $\left(\mathrm{S}_{29}\right)$ como esteranos mayoritarios (Figuras 4L y O). Otra diferencia se observó en el fragmentograma para el ion $\mathrm{m} / \mathrm{z}=$ 177 vinculado con los 25-norhopanos (desmetilhopanos), solo los crudos de la Formación Bajo Barreal presentaron estos biomarcadores (Figura 5).

Las proporciones pristano/fitano $(\mathrm{P} / \mathrm{F})$ fueron similares entre las muestras de la Formación Springhi11, estas oscilaron entre 2,59 y 1,94. En el caso de la Formación Pozo D-129 los crudos tuvieron cocientes cercanos a 1,00 (Tabla 1). Los valores del logaritmo en base 10 de las relaciones pristano/heptadecano $\left(\mathrm{P} / \mathrm{n}-\mathrm{C}_{17}\right)$ y fitano/octadecano $\left(\mathrm{F} / \mathrm{n}-\mathrm{C}_{18}\right)$ representados en la Figura 6A, se ubicaron en el límite de los intervalos definidos para la materia orgánica continental-mixta en el caso de los crudos CS1 y DM3. La muestra LM2 se posicionó ligeramente corrida hacia la zona mixta respecto de los otras dos antes mencionadas. En el caso de los crudos CO4 y RA5, se observó a la primera en el límite del intervalo marino-mixto y a la segunda levemente desplazada hacia la zona marina. La relación entre el n-nonacosano y el n-heptadecano $\left(\mathrm{n}-\mathrm{C}_{29} / \mathrm{n}-\mathrm{C}_{17}\right)$ mostró valores en un rango de 0,57 y 0,26 para las muestras bajo estudio (Tabla 1). Estos resultados indicarían crudos marinos, pero es importante destacar que esto solo aplica a las muestras de la Cuenca Austral ya que en los petróleos analizados de la Cuenca Golfo San Jorge existen evidencias de procesos biodegradables que han modificado la composición de estos (Peters et al., 2005).

Para las muestras bajo estudio se apreciaron contrastes importantes en el índice de gammacerano, ya que los crudos de la Formación Springhill dieron valores muy bajos y los de la Formación Bajo Barreal se situaron en torno al 13,0 \% (Tabla 1). Para acentuar todavía más las diferencias, cabe decir que los esteranos predominantes para Springhill fueron los coles$\operatorname{tanos}\left(\mathrm{S}_{27}\right)$ con un porcentaje alrededor del 50,0 \% y que la relación $\mathrm{D}_{27} / \mathrm{S}_{27}$ se ubicó por encima de uno. Respecto a la Formación Bajo Barreal destacaron los estigmastanos $\left(\mathrm{S}_{29}\right)$ con porcentajes en torno al 44,0\% y la relación para los diasteranos se posicionó por debajo de 0,50 (Tabla 1). Por otro lado, la representación de la relación dibenzotiofeno/fenantreno (DBT/Ph) vs pristano/fitano (P/F) determinó lutita marina como roca fuente de todos los crudos de la Cuenca Austral y a los de la Cuenca Golfo San Jorge en el límite entre carbonato y lutita marina (Figura 6B). 


\section{Discusión}

\section{Tipo de materia orgánica y biodegradación}

Los valores del logaritmo base 10 de las relaciones pristano/n-heptadecano $\left(\mathrm{P} / \mathrm{n}-\mathrm{C}_{17}\right)$ y fitano/n-octadecano $\left(\mathrm{F} / \mathrm{n}-\mathrm{C}_{18}\right)$ representados en la Figura $6 \mathrm{~A}$, junto a las distribuciones de n-alcanos en el fragmentograma para el ion $\mathrm{m} / \mathrm{z}=99$ y los valores de la relación $\mathrm{n}-\mathrm{C}_{29} / \mathrm{n}-\mathrm{C}_{17}$ entre 0,36 y 0,57 sugieren que la fuente orgánica de los crudos extraídos de la Formación Springhill es de tipo mixta, principalmente marina con una entrada secundaria continental (Tissot \& Welte, 1984). Estudios previos vinculados a esta formación indicaron que el petróleo se generó a partir de aportes episódicos de materia orgánica marina con aportes terrígenos (Cagnolatti et al., 1996). Además, el porcentaje relativo de colestanos $\left(\mathrm{S}_{27}\right)$ fue $50,0 \pm 5,0 \%$ para los crudos CS1, LM2 y DM3 lo cual respaldó los resultados antes mencionados, ya que estos compuestos se interpretan como derivados de fitoplancton y algas marinas (Peters et al., 2005). En otra investigación sobre crudos extraídos de Springhill llevados a cabo por Pittion y Goudaian (1992) se hizo énfasis en la naturaleza planctónica de la materia orgánica originaria de los mismos, dada a partir de los resultados que presentaron. Por otro lado, al analizar la presencia de hopanos se estableció una diferencia entre los crudos CS1 y LM2 respecto de DM3, ya que los primeros exhibieron una baja concentración de estos biomarcadores en relación con los segundos (Figura 4B, E y H). Trabajos previos realizados por Villar y Arbé (1993) manifestaron que la organofacies de la Formación Inoceramus Inferior (roca madre) asociada a Springhill se caracterizó por una entrada bacteriana inusualmente baja. La ausencia de desmetilhopanos en los fragmentogramas para el ion $\mathrm{m} / \mathrm{z}=177$ (Figura 5A), las relaciones $\mathrm{P} / \mathrm{n}-\mathrm{C}_{17}$ - $\mathrm{F} / \mathrm{n}-\mathrm{C}_{18}$ y el comportamiento de los n-alcanos implican que en los crudos CS1, LM2 y DM3 no hubo biodegradación. El petróleo de la Cuenca Austral se ha originado en rocas del Cretácico inferior, específicamente a partir de aportes de materia orgánica principalmente marina, con aportes secundarios continentales. El análisis de los hidrocarburos acumulados en sus reservorios llevado a cabo por
Cagnolatti y col. (1996), han indicado que procesos biodegradativos no se han producido sobre estos.

Al considerar las muestras de crudo primero debe tenerse en cuenta el fenómeno de biodegradación por el cual han atravesado las mismas, ya que esta transformación incide en gran medida sobre su composición y de manera indirecta sobre la caracterización del tipo de materia orgánica precursora (Peters et al., 2005). Esta afirmación se sostiene a partir de los fragmentogramas para el ion $\mathrm{m} / \mathrm{z}=177$ de los crudos CO4 y RA5 (Figura 5B), en los que se observó la presencia de desmetilhopanos asociados a una biodegradación severa (Peters et al., 2005). Además, existen diferencias entre ellos debido a que el patrón de $n$-alcanos en el fragmentograma del ion $\mathrm{m} / \mathrm{z}=99$ y las relaciones $\mathrm{P} / \mathrm{n}-\mathrm{C}_{17}-\mathrm{F} / \mathrm{n}-\mathrm{C}_{18}$ no parecen afectados por este proceso para el crudo $\mathrm{CO} 4$, pero sí para RA5. Esto sugiere que CO4 fue alterado por un proceso de degradación biológica severo que modificó sus propiedades primarias y luego se mezcló con nuevos aportes de crudo no alterados que enmascararon los efectos de la biodegradación original (Figari et al., 2002). Por su parte, la muestra RA5 también es una mezcla de petróleos, pero en la cual las nuevas entradas de hidrocarburos que resolubilizaron al crudo original experimentaron posteriormente un nuevo evento de degradación biológica. Los procesos de alteración en la Formación Bajo Barreal, especialmente la degradación biológica, han sido una constante en la historia de generación y acumulación de petróleos en la cuenca y su efecto ha tenido fuerte influencia en la calidad y tipo de petróleos producidos (Figari et al., 2002). En mayor detalle, Villar et al. (1996) documentaron los fundamentos de estos procesos de alteración, concluyendo que los hidrocarburos estudiados resultaron de procesos complejos de biodegradación y mezcla de activos a lo largo de extensos períodos del tiempo geológico. Las asociaciones de biomarcadores les indicaron que las acumulaciones responden a mezclas de petróleos intensamente biodegradados que han sido generados y alterados en una etapa temprana y subsecuentemente mezclados con petróleos frescos de nueva generación. Por otro lado, la suma de los porcentajes de ergostanos $\left(\mathrm{S}_{28}\right)$ y estigmastanos $\left(\mathrm{S}_{29}\right)$ constituyeron aproximadamente el 75,0\% de los esteranos regulares para los crudos extraídos de la Formación Bajo Barreal, lo cual su- 


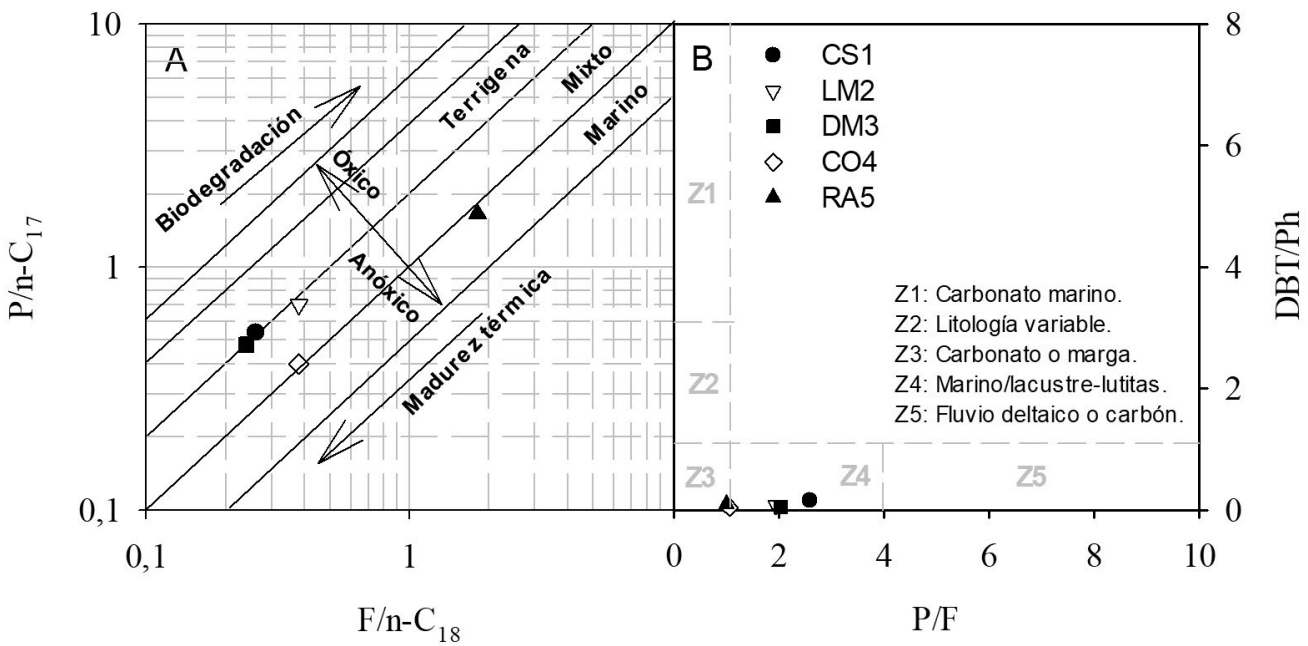

Figura 6.-A. Diagrama $\mathrm{P} / \mathrm{n}-\mathrm{C}_{17}$ en función de $\mathrm{F} / \mathrm{n}-\mathrm{C}_{18}$ en escala logarítmica, aplicado a la materia orgánica precursora, Shanmugam (1985). B. Diagrama "DBT/Ph" de Hughes et al. (1995) en función de P/F para determinar la litología de la roca generadora. En la Tabla 1 se desarrollan las ecuaciones matemáticas para el cálculo de las relaciones de diagnóstico presentadas en estas figuras.

giere un aporte de materia orgánica lacustre - continental en sus rocas generadoras (Peters et al., 2005). Los antecedentes de estudios geoquímicos como los publicados por Bellosi et al. (2002) describieron que la naturaleza de la materia orgánica sedimentada en la Formación Pozo D-129 (roca madre) fue predominantemente de tipo algal-lacustre con contribuciones continentales variables y el análisis visual del kerógeno indicó abundante material amorfo ocasionalmente acompañado de algas relacionadas con Botryococcus. Finalmente, en los fragmentogramas para el ion $\mathrm{m} / \mathrm{z}=191$ se observó una distribución tipo "V normal" para los terpanos tricíclicos $\mathrm{T}_{25} / \mathrm{T}_{24(4)} / \mathrm{T}_{26}$, los valores más altos para $\mathrm{T}_{23}, \mathrm{~T}_{24} \mathrm{y} \mathrm{T}_{25} \mathrm{y}$ el más bajo para $\mathrm{T}_{19}$. En un estudio publicado por Sylwan et al. (2008) se analizaron geoquímicamente mediante el uso de terpanos tri y tetracíclicos las tres unidades reconocidas como rocas madres de hidrocarburos de la cuenca: Formación Anticlinal Aguada Bandera, Formación Pozo Cerro Guadal y Formación Pozo D-129, y sólo está última presento el mismo patrón de terpanos tricíclicos que los crudos CO4 y RA5.

\section{Condiciones de sedimentación y litología de la roca madre}

La relación dibenzotiofeno/fenantreno (DBT/Ph) en función de pristano/fitano $(\mathrm{P} / \mathrm{F})$, es usada como indicador de ambiente depositacional de rocas se- dimentarias (Hughes et al., 1995). Las muestras estudiadas para la Cuenca Austral se ubicaron dentro de la zona cuatro en el diagrama de la Figura 6B, sugiriendo que las condiciones de sedimentación se asociaron a un paleoambiente de lutitas marinas, depositadas en condiciones de reducción moderada dado por valores de $\mathrm{P} / \mathrm{F}$ en torno a 2,0. La baja concentración de gammacerano $\left(\mathrm{G}_{30}\right)$ cercana al $0,0 \% \mathrm{y}$ valores de la relación $\mathrm{D}_{27} / \mathrm{S}_{27}$ superiores a 1,0 reflejan la ausencia de ambientes hipersalinos y el importante contenido de arcilla presente en la roca madre, respectivamente (Mello et al., 1988; Van Kaam-Peters et al., 1998). Pittion y Goudaian (1992) propusieron un modelo sedimentario en el cual las lutitas marinas de la Formación Inoceramus Inferior (roca madre) se depositaron en una capa de agua con baja presencia de oxígeno. En ese ambiente reductor la materia orgánica logró preservarse y permitió a esta secuencia sedimentaria desarrollar el potencial inicial para generar petróleo.

La materia orgánica precursora de los crudos de la Cuenca Golfo San Jorge sedimentó bajo condiciones reductoras soportado por valores de $\mathrm{P} / \mathrm{F}$ en torno a 1,0 o menores (Tabla 1). Además, CO4 y RA5 se posicionaron en el límite establecido para la zona tres (carbonato o marga) y cuatro (marino/lacustre-lutitas) de la Figura 6B. Es importante destacar que estos crudos se generaron en la Formación Pozo 
D-129, la cual es una roca madre carbonática asociada a un entorno anóxico (Figari et al., 2002; Villar et al., 1996). Finalmente, el índice de gammacerano mostró porcentajes superiores al 10,0 \% y la relación entre los diasteranos y esteranos de 27 átomos de carbono $\left(\mathrm{D}_{27} / \mathrm{S}_{27}\right)$ presentó valores menores a 0,50 para ambas muestras. Estos resultados son concluyentes con ambientes salobres en los cuales no destacan la presencia de arcillas (Villar et al., 1996).

\section{Conclusiones}

Los resultados obtenidos a partir de la caracterización del patrón de biomarcadores observado en las muestras estudiadas permiten sugerir que los crudos extraídos de la Formación Springhill - Cuenca Austral no presentaron indicios de biodegradación, además, se generaron a partir de kerógeno tipo II/ III (marino/continental) en una roca madre de tipo lutita marina bajo un ambiente con moderada concentración de oxígeno e importante presencia de minerales de arcillas. Por otro lado, los crudos captados de la Formación Bajo Barreal - Cuenca Golfo San Jorge fueron sometidos a intensos procesos de biodegradación. Estos se formaron a partir de materia orgánica algal-lacustre y contribución continental variable (Tipo I/II y Tipo II/III) sedimentados en la Formación Pozo D-129, roca generadora de tipo carbonática lacustre, bajo un ambiente salobre y reductor. De acuerdo con los resultados obtenidos se observó que los mismos concuerdan con los publicados anteriormente sobre crudos extraídos de estas formaciones y remarcan las disparidades que existen entre dos cuencas cercanas geográficamente, pero con historias geológicas muy diferentes. Además, los resultados y conclusiones alcanzados en el presente trabajo ponen de manifiesto la importancia de la geoquímica del petróleo como herramienta de prospección de hidrocarburos, en conjunción con otras disciplinas como la ingeniería del petróleo, la geología y la geofísica.

\section{AGRADECIMIENTOS}

Los autores agradecen la contribución financiera de la Universidad Tecnológica Nacional - Facultad Regional Santa Cruz (PI MSUTNSC0007712) y del Consejo Nacional de Investigaciones Científicas y Técnicas (CONICET). También agradecen al Ing.
Leandro Almonacid por su colaboración en la confección de la cartografía presentada, y al Ing. Mariano Bertinat, secretario de Estado de Ambiente de la Provincia de Santa Cruz, por su colaboración y gestión en la obtención de los permisos de muestreo de los yacimientos estudiados.

\section{Referencias}

Aramendia, I.; Ramos, M.E.; Geuna, S.; Cuitino, J.I. \& Ghiglione, M.C. (2018). A multidisciplinary study of the Lower Cretaceous marine to continental transition in the northern Austral-Magallanes basin and its geodynamic significance. Journal of South American Earth Sciences, 86: 54-69. https://doi.org/10.1016/j. jsames.2018.06.010

Barredo, S.; Chemale, F.; Marsicano, C.; Ávila, J.N.; Ottone, E.G. \& Ramos, V.A. (2012). Tectono-sequence stratigraphy and $\mathrm{U}-\mathrm{Pb}$ zircon ages of the Rincón Blanco Depocenter, northern Cuyo Rift, Argentina. Gondwana Research, 21 (2-3): 624-636. https:/doi. org/10.1016/j.gr.2011.05.016https://doi.org/10.1016/j. gr.2011.05.016

Bellosi, E.S.; Laffitte, G.A. \& Villar, H.J. (2002). Un nuevo sistema petrolero en el Oriente de la Cuenca del Golfo San Jorge: Revaloración de áreas marginales y exploratorias. V Congreso de Exploración y Desarrollo de Hidrocarburos, Mar del Plata, 85-92.

Cagnolatti, M.J.; Martins, R. \& Villar, H.J. (1996). La Formación Lemaire como probable generadora de hidrocarburos en el área Angostura, Provincia de Tierra del Fuego, Argentina. XIII Congreso Geológico Argentino y III Congreso de Exploración de Hidrocarburos, Buenos Aires, 123-139.

Cuitiño, J.I.; Varela, A.N.; Ghiglione, M.C.; Richiano, S. \& Poiré, G. (2019). The Austral-Magallanes Basin (Southern Patagonia): a synthesis of its stratigraphy and evolution. Latin American Journal of Sedimentology and Basin Analysis, 26 (2): 155-166.

Didyk, B.M.; Simoneit, B.R.T.; Brassell, S.C. \& Eglinton, G. (1978). Organic geochemical indicators of palaeoenvironmental conditions of sedimentation. Nature, 272: 216-222. https://doi.org/10.1038/272216a0

Figari, E.G.; Laffitte, G.; Lafourcade, P.; Strelkov, E.; Cid de la Paz, M. \& Villar, H.J. (1999). Los sistemas petroleros de la Cuenca del Golfo San Jorge: síntesis estructural, estratigráfica, y geoquímica. IV Congreso de Exploración y Desarrollo de Hidrocarburos, Mar del Plata, 1: 197-237.

Figari, E.G; Strelkov, E.; Paz, M.S.; Celaya, J. \& Laffitte, G. (2002). Cuenca del Golfo de San Jorge: síntesis estructural, estratigráfica y geoquímica. In: Geología y Recursos Naturales de Santa Cruz (M. J. Haller, Ed.). 
XV Congreso Geológico Argentino, El Calafate, 3: 571-601.

Gómez-Omil, R.; Arroyo, H.; Laffitte, G. \& Melo, A. (1990). Anteproyecto exploratorio para el Sector Oriental del Flanco Sur. Informe inédito, YPF S.A, Comodoro Rivadavia, $31 \mathrm{pp}$.

Hechem, J.J.; Homovc, J.F. \& Figari, E.G. (1990). Estratigrafía del Chubutiano (Cretácico) en la Sierra de San Bernardo, Cuenca del Golfo San Jorge, Chubut, Argentina. XI Congreso Geológico Argentino, San Juan, 3: 173-176.

Hughes, W.; Holba, A. \& Dzou, L. (1995). The ratios of dibenzotiophene to phenantrene and pristane to phytane as indicators of depositional environment and lithology of petroleum source rocks. Geochimica et Cosmochimica Acta, 59 (17): 3581-3598. https://doi. org/10.1016/0016-7037(95)00225-O

Hunt, J. (1996). Petroleum Geochemistry and Geology. Freeman, New York, 743 pp.

Killops, S.D. \& Killops, V.J. (2005). Introduction to Organic Geochemistry. Second Edition, Blackwell Publishing, 393 pp. https://doi.org/10.1002/9781118697214

Laffitte, G. \& Hechem J. (1993). Hydrocarbon generation and migration in the western Golfo San Jorge Basin. Third Latin American Congress on Organic Geochemistry, Extended abstracts, 69-71.

Laffitte, G.A.; Figari, E. \& Villar, H.J. (2001). Geochemical patterns and hydrocarbon habitat of the Golfo San Jorge Basin, Argentina. Hedberg Conference "New Technologies and New Play Concepts in Latin America", American Association of Petroleum Geologists, Mendoza, Argentina.

Leal, B.; Freites, M.; Bracho, E.; Martínez, M. \& López, L. (2011). Evaluación de la fase estacionaria en la separación SARA de dos crudos pertenecientes a la subcuenca de Barinas, Venezuela. Revista Técnica de la Facultad de Ingeniería Universidad del Zulia, 34 (3): 203-211.

Lerch, B.; Karlsen, D.; Seland, R. \& Backer-Owe, K. (2016). Depositional environment and age determination of oils and condensates from the Barents Sea. Petroleum Geoscience, 23: 190-209. https://doi. org/10.1144/petgeo2016-039

Mackenzie, A.; Brassell, S.; Eglington, G. \& Maxwell, J. (1982). Chemical fossils: The geological fate of steroids. Science, 217 (4559): 491-504. https://doi. org/10.1126/science. 217.4559 .491

Mello, M.R.; Telnaes, N.; Gaglianone, P.C.; Chicarelli, M.I.; Brassell, S.C. \& Maxwell, J.R. (1988). Organic geochemical characterisation of depositional palaeoenvironments of source rocks and oils in Brazilian marginal basins. Organic Geochemistry, 13 (1-3): 31-
45. https://doi.org/10.1016/0146-6380(88)90023-X

Moldowan, J.M.; Seifert, W.K. \& Gallegos, E.J. (1985). Relationship between petroleum composition and depositional environment of petroleum source rocks. Buletin of the American Association of Petroleum Geologist, 69 (8): 1255-1268. https://doi.org/10.1306/ AD462BC8-16F7-11D7-8645000102C1865D

Mpodozis, C.; Mella, P. \& Padva, D. (2011). Estratigrafía $\mathrm{y}$ megasecuencias sedimentarias en la cuenca Austral-Magallanes, Argentina y Chile. VIII Congreso de Exploración y Desarrollo de Hidrocarburos, Mar del Plata, 1: 97-137.

Peters, K.E. \& Moldowan J.M.; (1993). The Biomarker Guide. Interpreting Molecular Fossils in Petroleum and Ancient Sediments. Prentice Hall, New Jersey, 363 pp.

Peters, K.; Walters, C. \& Moldowan, J. (2005). The Biomarker Guide. Biomarkers and Isotopes in the Environment and Human History. Cambridge University Press, London, 492 pp.

Philp, P. (1985). Biological markers in fossil fuel production. Mass Spectrometric Reviews, 4 (1): 1-54. https:// doi.org/10.1002/mas.1280040102

Pittion, J. \& Goudaian, J. (1992). Source-rocks and oil generation in the Austral basin. XIII Word Petroleum Congress, Buenos Aires, 2: 113-120.

Ramos, V.A. (2015). Evolución de la Cuenca Golfo San Jorge: su estructuración y régimen tectónico. Revista de la Asociación Geológica Argentina, 72 (1): 12 -20.

Ramos, M.E.; Suárez, R.; Boixart, G.; Ghiglione, M. \& Ramos, V.A. (2019). The structure of the northern Austral Basin: Tectonic inversion of Mesozoic normal faults. Journal of South American Earth Sciences, 94. https://doi.org/10.1016/j.jsames.2019.05.013

Richiano, S.; Varela, A.N. \& Poire, D.G. (2016). Heterogeneous distribution of trace fossils across initial transgressive deposits in rift basins: an example from the Springhill Formation, Argentina. Lethaia, 49 (4): 524-539. https://doi.org/10.1111/let.12163

Schiuma, M.; Hinterwimmer, G. \& Vergani, G. (2018). Rocas Reservorio de las Cuencas Productivas de la Argentina. IAPG, Buenos Aires, 1006 pp.

Seifert, W. \& Moldowan J. (1981). Palaeoreconstruction by biological markers. Geochimica et Cosmochimica Acta, 45: 783-794. https://doi.org/10.1016/00167037(81)90108-3

Seifert, W.K.; Moldowan, J.M. \& Demaison, G.J. (1984). Source correlation of biodegraded oils. Organic Geochemistry, 6: 633-643. https://doi.org/10.1016/01466380(84)90085-8

Shanmugam, G. (1985). Significance of coniferous rain forests and related organic matter in generating commercial quantities of oil, Gippsland basin, Australia. 
Bulletin of the American Association of Petroleum Geologist, 69 (8): 1241-1254. https://doi.org/10.1306/ AD462BC3-16F7-11D7-8645000102C1865D

Sinninghe-Damste, J.; Kenig, F.; Koster, J. \& Leeuw, J. (1995). Evidence for gammacerano as an indicator of water column stratification. Geochimica et Cosmochimica Acta, 59: 1895-1900. https://doi. org/10.1016/0016-7037(95)00073-9

Speers, G.C. \& Whitehead E.V. (1969). Crude petroleum. In: Organic Geochemistry: Methods and Results (Eglinton, G. \& Murphy M.T.J., Eds.), Springer-Verlag, Berlin, pp. 638-702. https://doi.org/10.1007/9783-642-87734-6 34

Stashenko, E.; Martínez, J. \& Robles, M. (2014). Extracción selectiva y detección específica de biomarcadores saturados del petróleo. Scientia Chromatographica, 6 (4): 251-268. https://doi.org/10.4322/sc.2015.010

Sylwan, C.A. (2001). Geology of the Golfo San Jorge Basin, Argentina. Journal of Iberian Geology, 27: 123-157.

Sylwan, C.A.; Rodriguez, J.F. \& Strelkov, E.E. (2008). Petroleum Systems of The Golfo San Jorge Basin, Argentina. VII Congreso de Exploración y Desarrollo de Hidrocarburos (Simposio de Sistemas Petroleros de las Cuencas Andinas), European Association of Geoscientists \& Engineers, Conference Proceedings. https://doi.org/10.3997/2214-4609-pdb.266.4

Tissot, B. \& Welte, D. (1984). Petroleum formation and occurrence. Springer-Verlag, Berlín, 666 pp. https:// doi.org/10.1007/978-3-642-87813-8
Tomas, G.J.; Vargas, W. \& Acuña, A.J. (2020). Evaluación geoquímica por biomarcadores del yacimiento Del Mosquito de la Cuenca Austral de la Patagonia Argentina. Revista de la Sociedad Geológica de España, 33 (2): 31-40.

Van Kaam-Peters, H.M.E.; Koster, J.; Van Der Gaast, S.J.; Dekker, M.; De Leeuw, J.W. \& Sinninghe Damste, J.S. (1998). The effect of clay minerals on diasterane/sterane ratios. Geochimica et Cosmochimica Acta, 62 (17): 2923-2929. https://doi.org/10.1016/S00167037(98)00191-4

Villar, H.J. \& Arbe, H.A. (1993). Oil generation in the Esperanza Area, Austral Basin, Argentina. En: III Latin American Congress on Organic Geochemistry (Mello, M.R. \& Trindade, L.A.F., Eds.), Manaus, 150-153 pp.

Villar, H.J.; Sylwan, C.; Gutiérrez Pleimling, A.; Miller, M.; Castaño, J. \& Dow, W. (1996). Formación de petróleos pesados a partir de procesos de biodegradación y mezcla en el sistema petrolero Pozo D-129-Cañadón Seco, Flanco Sur de la Cuenca del Golfo San Jorge, Provincia de Santa Cruz, Argentina. XIII Congreso Geológico Argentino y III Congreso de Exploración de Hidrocarburos, Buenos Aires, 45-60 pp.

Zerfass, H.; Ramos, V.A.; Ghiglione, M.C.; Naipauer, M.; Belotti, H.J. \& Carmo, I.O. (2017). Folding, thrusting and development of push-up structures during the Miocene tectonic inversion of the Austral basin, southern patagonian Andes (50 ${ }^{\circ} \mathrm{S}$ ). Tectonophysics, 699: 102120. https://doi.org/10.1016/j.tecto.2017.01.010 